\title{
Saúde, trabalho e envelhecimento no Brasil
}

\author{
Health, work, and aging in Brazil
}

Luana Giatti 1

Sandhi M. Barreto ${ }^{1}$

2 Núcleo de Estudos em Saúde Pública e Envelhecimento, Centro de Pesquisas René Rachou, Fundação Oswaldo Cruz/ Universidade Federal de Minas Gerais Av. Augusto de Lima 1715, Belo Horizonte, $M G$ 30190-002, Brasil.

\begin{abstract}
The rapid aging process of the Brazilian population is accompanied by a similar change in the composition of the country's work force. The objective of this study is to determine health differentials in the elderly according to their insertion in the work market, after considering the influence of socio-demographic factors. This study included 2,886 males $\geq 65$ years residing in ten Brazilian metropolitan areas and included in the National Household Survey conducted by the National Institute of Geography and Statistics, or National Census Bureau (IBGE) in 1998. The analysis included the chi-square and odds ratios estimated by multiple logistic regression. More than a fourth of the elderly worked. Among the formally retired elderly, those who still worked were younger seniors, those with more schooling, and those with higher per capita family income; they reported fewer chronic diseases and presented less difficulty in performing their activities of daily living, but did not show any differences in relation to health services utilization. According to our results, health and especially indicators of autonomy and physical mobility are independent predictive factors for the elderly to remain active.
\end{abstract}

Key words Aging Health; Aging; Work

Resumo O envelhecimento da população tem importante reflexo na composição etária da população economicamente ativa. O objetivo do presente estudo é determinar os diferenciais de saúde dos idosos, segundo sua inserção no mercado de trabalho, após considerar a influência dos fatores sócio-demográficos. Este trabalho incluiu 2.886 idosos do sexo masculino, com 65 anos ou mais de idade, residentes em dez regiões metropolitanas brasileiras e participantes da Pesquisa Nacional por Amostra de Domicílios, Fundação Instituto Nacional de Geografia e Estatística realizada em 1998. A análise incluiu o qui-quadrado e a odds ratio estimado através de regressão logística múltipla. Mais de um quarto dos idosos trabalhava. Em relação aos aposentados, os que trabalhavam eram mais jovens, tinham maior escolaridade e maior renda domiciliar per capita; eles relataram menor freqüência de doenças crônicas, apresentaram menor dificuldade para realização das atividades da vida diária, mas não apresentaram diferenças com relação ao uso de serviços de saúde. Nossos resultados indicam que a saúde e em especial os indicadores de autonomia e mobilidade física são fatores preditivos independentes da permanência na vida ativa em idades mais elevadas.

Palavras-chave Saúde do Idoso; Envelhecimento; Trabalho 


\section{Introdução}

O envelhecimento da população é um fenômeno mundial. Nos países desenvolvidos, esse processo se deu lentamente, em uma situação de evolução econômica, de crescimento do nível de bem-estar e redução das desigualdades sociais (Moreira, 1998). Nos anos mais recentes, esse processo ganha maior importância nos países em desenvolvimento, com o aumento acelerado da população de sessenta anos e mais em relação à população geral. Aumentos de até $300 \%$ da população idosa são esperados nesses países, especialmente na América Latina (Truelsen et al., 2001).

O Brasil apresenta um dos mais agudos processos de envelhecimento populacional entre os países mais populosos. A proporção de pessoas idosas com sessenta anos e mais aumentou de $6,1 \%$ (7.204.517 habitantes), em 1980, para 8,6\% (14.536.029 habitantes) em 2000, correspondendo a um aumento absoluto de 7,3 milhões de indivíduos (IBGE, 1981, 2001). A razão de dependência demográfica (soma da população menor de 15 anos e de 65 e mais anos dividida pela população com 15 a 64 anos) hoje, definida principalmente pelo segmento jovem, será modificada para uma dependência idosa ao final de 2.050 (Moreira, 1998).

Uma importante conseqüência dessa transição demográfica é o envelhecimento da população ativa. Nos países onde essa transição já ocorreu, a população em idade ativa é mais envelhecida, e esse processo tende a acentuar-se com o tempo (Costa, 2000; Schkolnik, 1989). No Brasil, Wajnman (1997), estudando as perspectivas de crescimento da população economicamente ativa (PEA), identifica que o segmento dessa população que mais cresceu no período entre 1980 e 1990 foi o de 25 a 49 anos, e entre 2000 e 2020 será o segmento correspondente às pessoas acima de cinqüenta anos, confirmando a tendência de envelhecimento da PEA.

Inúmeros estudos têm demonstrado que as pessoas que trabalham apresentam melhores condições de saúde do que a população geral, e que as pessoas doentes e incapazes são, geralmente, excluídas do mercado de trabalho. $\mathrm{O}$ desemprego tem sido associado a piores condições de saúde, a maiores taxas de mortalidade e maior prevalência de sintomas psiquiátricos, de hipertensão arterial e hábitos de vida nocivos, como consumo de bebida alcoólica e cigarro (Arber, 1987; Janlert, 1997). Na realidade, o trabalho é descrito como fator seletivo e também positivo para a preservação de uma melhor condição de saúde, constituindo um importante viés em estudos epidemiológicos ocupacionais, quando populações trabalhadoras são comparadas à população geral (Carpenter, 1990). Em estudos longitudinais, esse viés é mais fortemente observado para doenças que se manifestam precocemente do que para as doenças crônico-degenerativas de aparecimento mais tardio, como as neoplasias.

A associação entre saúde e trabalho está bem documentada em países desenvolvidos, especialmente entre os adultos e jovens (Checkoway et al., 1989). Com o envelhecimento da população trabalhadora, e dadas as necessidades específicas de saúde da população idosa (LimaCosta, 2000), alguns autores têm apontado a importância de investigar as desigualdades em saúde entre os idosos através da qualificação do trabalho. Em um desses estudos, Marmot \& Shipley (1996) identificaram uma associação entre menor qualificação no trabalho e maior morbidade e mortalidade. Esta associação seria explicada pela relação direta entre qualificação ocupacional e escolaridade, renda, auto-estima e condições de vida de um modo geral. Na verdade, a simples situação de estar trabalhando tem sido apontada como um potente e independente fator preditivo de maior sobrevida (Blanc et al., 1994).

Pouco se sabe sobre os determinantes da permanência na vida ativa em idades mais avançadas em países em desenvolvimento como o Brasil. Estudos sobre a mortalidade em população adulta indicam que o viés do trabalhador sadio é mais acentuado no Brasil que em países desenvolvidos (Barreto et al., 1996). Não sabemos, entretanto, se as desigualdades em saúde relacionadas à inserção no mercado de trabalho, observadas entre os adultos, repetem-se ou mesmo acentuam-se em idades mais elevadas, já que o envelhecimento é caracterizado pelo aumento da prevalência de doenças e maior uso de serviços de saúde.

O objetivo do presente estudo é determinar os diferenciais de saúde entre homens idosos, segundo sua condição de inserção no mercado de trabalho, após considerar a influência dos fatores sócio-demográficos.

\section{Metodologia}

\section{População do estudo}

A população deste estudo corresponde a indivíduos do sexo masculino residentes nas regiões metropolitanas de Belém, Fortaleza, Recife, Salvador, Belo Horizonte, São Paulo, Rio de Janeiro, Curitiba, Porto Alegre e Distrito Federal, com idade igual ou superior a 65 anos, participantes 
da Pesquisa Nacional por Amostra de Domicílios (PNAD) realizada em 1998.

\section{- Fonte de dados}

A PNAD foi conduzida no período de 20 a 26 de setembro de 1998 pela Fundação Instituto Brasileiro de Geografia e Estatística (IBGE). É um inquérito populacional de âmbito nacional, representativo da população. A PNAD é realizada anualmente e tem como objetivo obter informações sobre características sócio-demográficas, de habitação, educação, trabalho e rendimento. No ano referido, também, foram investigados aspectos referentes à morbidade, cobertura por planos de saúde, acesso e utilização dos serviços de saúde para toda a população, além de características de mobilidade física dos moradores de 14 anos ou mais. Na PNAD, as informações são obtidas por meio de entrevista com um informante nos domicílios selecionados.

\section{- A amostragem}

A amostra utilizada na PNAD é obtida em três estágios de seleção. O primeiro estágio consiste na seleção dos municípios. Estes foram classificados em duas categorias: os auto-representativos, com probabilidade um de pertencer à amostra, e os não auto-representativos, os quais foram separados em estratos e selecionados com reposição e com probabilidade proporcional à população residente em cada município, obtida no censo de 1991. Nesses dois estratos de municípios, foi selecionada uma amostra com reposição de setores censitários, com probabilidade proporcional ao número de domicílios existentes no censo de 1991 em cada setor, o que vem a corresponder ao segundo estágio de seleção. No último estágio da amostra, os domicílios foram selecionados por setor censitário, sendo investigadas as informações sobre todos os residentes de cada um.

No presente estudo, foram utilizados apenas os municípios das regiões metropolitanas, todos eles com probabilidade um de pertencer à amostra.

\section{- Variáveis do estudo}

A variável dependente deste estudo foi a situação no mercado de trabalho, composta de três categorias independentes: aposentado, trabalhando e outra condição. Os idosos ocupados foram classificados como trabalhando, independentemente de receberem ou não aposentadoria; os que eram aposentados e não traba- lhavam foram classificados como aposentados e os que não estavam ocupados e não recebiam aposentadoria foram classificados como outra condição. Os desempregados, ou seja, aqueles que procuravam por trabalho e não eram aposentados não foram considerados neste estudo pelo reduzido número de observações identificadas (12 idosos). Da mesma forma, devido ao pequeno número de idosos na categoria outra condição $(n=115)$, a presente análise se restringiu à comparação entre os que trabalhavam e o grupo aposentado.

As características sócio-demográficas estudadas foram idade em anos (65-69, 70-74, +75 anos), escolaridade em anos de estudos (0-3, 47, 8-10, 11+ anos), renda domiciliar per capita, obtida dividindo-se a renda domiciliar pelo número de moradores e agrupada em quintis, condição no domicílio (pessoa de referência, sim ou não) e região metropolitana de residência. Essas regiões foram agregadas segundo as macrorregiões de residência: Sudeste (regiões metropolitanas de São Paulo, Rio de Janeiro e Belo Horizonte), Sul (regiões metropolitanas de Curitiba e Porto Alegre), Nordeste (regiões metropolitanas de Fortaleza, Recife e Salvador), Norte (região metropolitana de Belém) e a região metropolitana do Distrito Federal.

Os indicadores de saúde utilizados neste estudo foram percepção da própria saúde, afastamento de quaisquer das atividades habituais nas últimas duas semanas, ter estado acamado nas últimas duas semanas e relato de doença crônica. A percepção da própria saúde foi obtida através da questão "De um modo geral, considera seu próprio estado de saúde como”, com as opções "muito bom, bom, regular, ruim e muito ruim" para resposta, que foram agrupadas em três estratos: muito bom/bom, regular e muito ruim/ruim. Foi considerado, também, um último estrato (outra pessoa) na construção da variável, quando essa informação não foi obtida do próprio indivíduo. Isto foi feito devido aos aspectos subjetivos envolvidos na percepção da própria saúde que só podem ser apreendidos nos casos em que o próprio indivíduo responde a entrevista. A informação sobre afastamento de quaisquer das atividades habituais nas últimas duas semanas (sim ou não) foi obtida através da pergunta "Nas duas últimas semanas deixou de realizar quaisquer de suas atividades habituais por motivo de saúde?" e sobre ter estado acamado também nas últimas duas semanas ( duas últimas semanas esteve acamado?”. A variável relato de doença crônica foi constituída com base no relato de uma ou mais das seguintes condições: artrite ou reumatismo, câncer, 
diabetes, bronquite ou asma, hipertensão, doença do coração, doença renal crônica e cirrose, transformadas em uma única variável dicotômica, presença ou ausência de doença crônica. A utilização dos serviços de saúde foi estudada por meio das seguintes variáveis: consulta médica e consulta odontológica nas duas últimas semanas, ambas obtidas através da pergunta "Qual foi o principal atendimento de saúde que recebeu?"; última visita ao dentista medida em anos $(<1,1-2,+3$ e nunca consultou) e internação hospitalar no último ano (sim ou não). A cobertura por plano privado de saúde (sim ou não) também foi contemplada neste trabalho.

No presente estudo, os indicadores de autonomia e mobilidade física foram estudados mediante o grau de dificuldade relatado para realizar as seguintes tarefas: alimentar-se, tomar banho ou ir ao banheiro; correr, levantar objetos pesados, praticar esportes ou realizar trabalhos pesados; empurrar mesa ou realizar consertos domésticos, subir ladeira ou escada; abaixar-se, ajoelhar-se ou curvar-se; andar mais de um quilômetro; andar cerca de 100 metros. Todos esses indicadores foram obtidos através da questão "Normalmente, por problema de saúde, tem dificuldade para...", com as opções "não consegue, tem grande dificuldade, tem pequena dificuldade, não tem dificuldade" para resposta. $\mathrm{Pa}-$ ra análise, as respostas foram estratificadas em sem dificuldade, com alguma dificuldade e com grande dificuldade ou não consegue.

\section{- Análise dos dados}

A presente análise foi restrita aos homens em virtude das especificidades que determinam a inserção de cada gênero no mercado de trabalho.

A análise da associação entre a situação no mercado de trabalho e características sócio-demográficas, indicadores de saúde, de utilização dos serviços de saúde e de autonomia e mobilidade física foi baseada no qui-quadrado de Pearson (Armitage \& Berry, 1987), na odds ratio bruta e seu intervalo de confiança (Schlesslman, 1982).

A regressão logística múltipla (Hosmer \& Lamershow, 1989) foi utilizada para investigar a associação independente entre a situação no mercado de trabalho e cada um dos indicadores de saúde, de utilização de serviços de saúde e de autonomia e mobilidade física incluídos no estudo. Aposentado foi a categoria de referência na análise por ser o estrato com maior número de pessoas. Na análise multivariada, cada variável de interesse foi ajustada pela idade, escolaridade, renda domiciliar per capita, pessoa de referência no domicílio e macrorregião de residência.

O programa STATA foi usado para a análise dos dados, uma vez que dispõe de procedimentos para análise de inquéritos amostrais complexos. Estes procedimentos permitem incorporar os pesos distintos das observações que influenciam as estimativas pontuais de parâmetros da população total. Na presente análise, foi incluído o peso amostral através da variável "Peso da Pessoa" (v4729), que equivale ao inverso da fração amostral. Valendo-se das variáveis UF (unidade da federação), UPA (delimitação do município) e $v 4602$ (strata), foi criada uma nova variável seguindo algoritmo fornecido pela IBGE para corrigir o efeito do desenho amostral (F. Viacava, comunicação pessoal). A incorporação desta nova variável na análise permite obter estimativas aproximadas mais corretas do erro padrão, uma vez que este é influenciado também pela conglomeração das unidades e estratificação derivadas do desenho amostral de múltiplos estágios da PNAD (Pessoa et al., 1997).

\section{Resultados}

Dos 2.898 idosos selecionados, 2.886 participaram desse estudo e 12 indivíduos foram excluídos porque não se enquadraram nas categorias da variável dependente considerada neste estudo. Entre os participantes, a mediana das idades foi 72,27 .

A distribuição proporcional da população estudada de acordo com a situação no mercado de trabalho mostra que a maioria dos homens $(68,46 \%)$ estava aposentada, $26,9 \%$ trabalhavam e apenas 4,6\% não trabalhavam e nem eram aposentados. Estes últimos não foram considerados na análise da associação entre as variáveis independentes e a situação no mercado de trabalho.

Entre os idosos ocupados, $64 \%$ realizavam trabalho informal, ou seja, não tinham carteira assinada nem contribuíam para a previdência social; $66 \%$ tinham uma jornada de trabalho igual ou maior do que 40 horas semanais; maior proporção (39\%) trabalhava por conta própria, $29,3 \%$ eram empregados, $13,9 \%$ eram empregadores, 11,8\% faziam trabalhos não remunerados, $3,7 \%$ eram funcionários públicos e 2,3\% realizavam trabalhos domésticos. Os ramos de atividade econômica que concentraram as maiores proporções de idosos trabalhando foram os de prestação de serviço $(18,2 \%)$, comércio $(15,4 \%)$, indústria da construção (11,3\%), atividade agrícola $(10,8 \%)$, setor social $(10,3 \%)$ e indústria da transformação $(8,3 \%)$. A renda pessoal mensal 
dos idosos que trabalhavam apresentou uma mediana de $\mathrm{R} \$ 610,00$, enquanto a mediana dos aposentados foi de $\mathrm{R} \$ 350,00$.

A distribuição percentual dos fatores sóciodemográficos, dos indicadores de saúde, de utilização dos serviços de saúde e de autonomia e mobilidade física, segundo a situação no mercado de trabalho, é mostrada nas Tabelas $1,2 \mathrm{e}$ 3 . Entre os homens que trabalhavam, a maioria tinha idade entre 65 e 69 anos, 25,7\% tinham 11 ou mais anos de estudo, $90 \%$ eram pessoas de referência no domicílio e $27,6 \%$ tinham renda domiciliar per capita maior ou igual a $\mathrm{R} \$ 680,00$ (Tabela 1).

Em relação à pergunta sobre a percepção da própria saúde, $25,9 \%$ e $24,4 \%$ dos aposentados e dos que trabalhavam, respectivamente, relataram a saúde como muito boa/boa. Menor proporção de idosos que trabalhavam relatou afastamento das atividades habituais e ter estado acamado nas duas últimas semanas, assim como menor proporção de doença crônica, de consulta médica nas duas últimas semanas e de internação hospitalar no último ano. Maior proporção de idosos que trabalhavam relatou cobertura por plano de saúde privado, consulta odontológica nas duas últimas semanas e menor tempo desde a última visita ao dentista (Tabela 2). As doenças crônicas prevalentes entre os que trabalhavam e os que estavam aposentados foram hipertensão arterial (41\% e 33\%), artrite/reumatismo (23,8\% e $20 \%)$ e doença do coração (23\% e 15\%). Hipertensão, doença do coração e câncer foram significativamente menos freqüentes entre os que trabalhavam ( $\mathrm{p}=0,0016, \mathrm{p}<0,0001 \mathrm{e} \mathrm{p}=0,0011)$.

Entre os indivíduos que trabalhavam, havia maior proporção de idosos que não relataram dificuldade para realização das atividades indicadoras de autonomia e mobilidade física investigados na PNAD (Tabela 3 ).

$\mathrm{Na}$ análise univariada, o fato de estar trabalhando esteve inversamente associado com a idade, diretamente associado com a escolaridade, com renda domiciliar per capita, com o fato de ser pessoa de referência no domicílio e morar na Região Sul do país (Tabela 4).

Na Tabela 5, são apresentados as odds ratios brutas e ajustadas da associação entre a situação no mercado de trabalho e os indicadores de saúde e de utilização de serviços. Na análise univariada, os seguintes indicadores estiveram associados com a permanência no trabalho: menor freqüência de afastamento das atividades habituais nas duas últimas semanas e de doenças crônicas, maior relato de consulta odontológica nas duas últimas semanas e menor tempo desde a última visita ao dentista. Após ajustamento

\begin{tabular}{|c|c|c|}
\hline \multicolumn{3}{|c|}{$\begin{array}{l}\text { Distribuição proporcional da população idosa masculina residente em regiões } \\
\text { metropolitanas de acordo com a situação no mercado de trabalho e características } \\
\text { sócio-demográficas. Brasil, } 1998 \text {. }\end{array}$} \\
\hline & \multicolumn{2}{|c|}{ Situação no mercado de trabalho } \\
\hline & Aposentado $(n=1.963)$ & Trabalhando $(n=808)$ \\
\hline \multicolumn{3}{|l|}{ Idade em anos } \\
\hline $65-69$ & 35,36 & 57,35 \\
\hline $70-74$ & 27,69 & 28,61 \\
\hline \multirow[t]{2}{*}{$75+$} & 36,85 & 13,77 \\
\hline & $p<0,0001$ & \\
\hline \multicolumn{3}{|l|}{ Escolaridade em anos } \\
\hline $0-3$ & 38,62 & 38,11 \\
\hline $4-7$ & 33,71 & 27,02 \\
\hline $8-10$ & 9,99 & 8,96 \\
\hline \multirow[t]{2}{*}{$>11$} & 17,65 & 25,72 \\
\hline & $p=0,0008$ & \\
\hline \multicolumn{3}{|c|}{ Pessoa de referência no domicílio } \\
\hline Sim & 83,22 & 90,02 \\
\hline \multirow[t]{2}{*}{ Não } & 16,78 & 9,98 \\
\hline & $p<0,0001$ & \\
\hline \multicolumn{3}{|c|}{ Renda domiciliar per capita (em R\$) } \\
\hline$<130,00$ & 24,60 & 18,61 \\
\hline $130,00 \vdash 201,43$ & 16,44 & 13,56 \\
\hline $201,43 \vdash 341,00$ & 21,25 & 21,91 \\
\hline $341,00 \vdash 680,00$ & 21,45 & 18,35 \\
\hline \multirow[t]{2}{*}{$\geq 680,00$} & 16,26 & 27,57 \\
\hline & $p=0,0010$ & \\
\hline \multicolumn{3}{|l|}{ Região de residência } \\
\hline Sudeste & 70,73 & 66,50 \\
\hline Sul & 10,61 & 13,92 \\
\hline Nordeste & 14,76 & 15,72 \\
\hline Norte & 1,54 & 1,53 \\
\hline \multirow[t]{2}{*}{ Distrito Federal } & 2,35 & 2,33 \\
\hline & $p=0,0098$ & \\
\hline
\end{tabular}

Fonte: PNAD 1998.

Valor p: teste Qui-quadrado de Pearson, com correção de Rao \& Scott. As proporções levam em consideração o peso amostral dos indivíduos. Foram excluídos os indivíduos sem trabalho ou aposentadoria, que representaram apenas $5 \%$ da população estudada.

pelas características sócio-demográficas, apenas o relato de doenças crônicas manteve-se negativo e estatisticamente associado ao trabalho.

Os resultados da análise univariada da associação entre a situação no mercado de trabalho e os indicadores de autonomia e mobilidade física apontam que, comparados aos aposentados, os idosos que trabalhavam tiveram menor dificuldade para realizar todas as atividades relacionadas à autonomia e mobilidade física estudadas, com um gradiente negativo na associação com todas essas variáveis. Essas associa- 
Distribuição proporcional da população idosa masculina residente em regiões metropolitanas de acordo com a situação no mercado de trabalho e indicadores de saúde e utilização de serviços de saúde. Brasil, 1998.

\begin{tabular}{|c|c|c|}
\hline & \multicolumn{2}{|c|}{ Situação no mercado de trabalho } \\
\hline & Aposentado $(n=1.963)$ & Trabalhando $(n=808)$ \\
\hline \multicolumn{3}{|c|}{ Percepção da própria saúde } \\
\hline Muito boa/boa & 25,92 & 24,41 \\
\hline Regular & 21,04 & 18,00 \\
\hline Muito ruim/ruim & 7,27 & 4,65 \\
\hline \multirow[t]{2}{*}{ Outra pessoa } & 45,78 & 52,94 \\
\hline & $p=0,0588$ & \\
\hline \multicolumn{3}{|c|}{$\begin{array}{l}\text { Afastamento das atividades habituais nas } \\
\text { duas últimas semanas }\end{array}$} \\
\hline Sim & 13,48 & 8,85 \\
\hline \multirow[t]{2}{*}{ Não } & 86,52 & 91,15 \\
\hline & $p=0,0216$ & \\
\hline \multicolumn{3}{|c|}{ Acamado nas duas últimas semanas } \\
\hline Sim & 8,98 & 6,40 \\
\hline \multirow[t]{2}{*}{ Não } & 91,02 & 93,60 \\
\hline & $p=0,1130$ & \\
\hline \multicolumn{3}{|c|}{ Relato de doença crônica1 } \\
\hline Sim & 64,46 & 57,02 \\
\hline \multirow[t]{2}{*}{ Não } & 35,54 & 42,79 \\
\hline & $p=0,0237$ & \\
\hline \multicolumn{3}{|c|}{ Plano de saúde privado } \\
\hline Sim & 42,17 & 44,99 \\
\hline \multirow[t]{2}{*}{ Não } & 57,83 & 55,01 \\
\hline & $p=0,5246$ & \\
\hline \multicolumn{3}{|c|}{ Consulta médica nas duas últimas semanas } \\
\hline Sim & 18,05 & 15,99 \\
\hline \multirow[t]{2}{*}{ Não } & 81,95 & 84,01 \\
\hline & $p=0,4302$ & \\
\hline \multicolumn{3}{|c|}{ Consulta odontológica nas duas últimas semanas } \\
\hline Sim & 0,45 & 2,01 \\
\hline \multirow[t]{2}{*}{ Não } & 99,55 & 97,99 \\
\hline & $p=0,0010$ & \\
\hline \multicolumn{3}{|c|}{ Última visita ao dentista (anos) } \\
\hline$<1$ & 15,28 & 24,88 \\
\hline $1-2$ & 11,51 & 13,12 \\
\hline $3+$ & 69,69 & 58,91 \\
\hline \multirow[t]{2}{*}{ Nunca } & 3,52 & 3,09 \\
\hline & $p<0,0001$ & \\
\hline \multicolumn{3}{|c|}{ Internação hospitalar no último ano } \\
\hline Sim & 14,09 & 11,56 \\
\hline \multirow[t]{2}{*}{ Não } & 85,91 & 88,44 \\
\hline & $p=0,1184$ & \\
\hline
\end{tabular}

Fonte: PNAD 1998.

Valor p: teste Qui-quadrado de Pearson, com correção de Rao \& Scott.

As proporções levam em consideração o peso amostral dos indivíduos. Foram excluídos os indivíduos sem trabalho ou aposentadoria, que representaram apenas $5 \%$ da população estudada.

1 Doenças crônicas consideradas: artrite ou reumatismo, câncer, diabetes, bronquite ou asma, hipertensão, doença do coração e cirrose. 
Distribuição proporcional da população idosa masculina residente em regiões metropolitanas, de acordo com a situação no mercado de trabalho e indicadores de autonomia e mobilidade física. Brasil, 1998.

\begin{tabular}{|c|c|c|}
\hline & \multicolumn{2}{|c|}{ Situação no mercado de trabalho } \\
\hline & Aposentado $(n=1.963)$ & Trabalhando $(n=808)$ \\
\hline \multicolumn{3}{|c|}{$\begin{array}{l}\text { Dificuldade para alimentar-se, tomar banho } \\
\text { ou ir ao banheiro }\end{array}$} \\
\hline Sem dificuldade & 82,02 & 93,56 \\
\hline Alguma dificuldade & 9,17 & 4,24 \\
\hline Grande dificuldade & 8,81 & 1,82 \\
\hline \multirow[t]{2}{*}{ Sem informação } & 0,0 & 0,38 \\
\hline & $p<0,0001$ & \\
\hline \multicolumn{3}{|c|}{$\begin{array}{l}\text { Dificuldade para correr, levantar objeto pesado, } \\
\text { praticar esporte ou realizar trabalho pesado }\end{array}$} \\
\hline Sem dificuldade & 23,68 & 45,04 \\
\hline Alguma dificuldade & 24,06 & 20,42 \\
\hline Grande dificuldade & 43,44 & 32,72 \\
\hline \multirow[t]{2}{*}{ Sem informação } & 8,81 & 1,82 \\
\hline & $p<0,0001$ & \\
\hline \multicolumn{3}{|c|}{$\begin{array}{l}\text { Dificuldade para empurrar mesa ou realizar } \\
\text { conserto doméstico }\end{array}$} \\
\hline Sem dificuldade & 45,13 & 74,06 \\
\hline Alguma dificuldade & 23,66 & 14,72 \\
\hline Grande dificuldade & 22,40 & 9,40 \\
\hline \multirow[t]{2}{*}{ Sem informação } & 8,81 & 1,82 \\
\hline & $p<0,0001$ & \\
\hline \multicolumn{3}{|c|}{ Dificuldade para subir ladeira ou escada } \\
\hline Sem dificuldade & 32,14 & 55,26 \\
\hline Alguma dificuldade & 27,62 & 27,25 \\
\hline Grande dificuldade & 31,43 & 15,68 \\
\hline \multirow[t]{2}{*}{ Sem informação } & 8,81 & 1,82 \\
\hline & $p<0,0001$ & \\
\hline \multicolumn{3}{|c|}{ Dificuldade para abaixar-se, ajoelhar-se ou curvar-se } \\
\hline Sem dificuldade & 35,54 & 58,48 \\
\hline Alguma dificuldade & 25,66 & 22,38 \\
\hline Grande dificuldade & 29,91 & 17,32 \\
\hline \multirow[t]{2}{*}{ Sem informação } & 8,89 & 1,82 \\
\hline & $p<0,0001$ & \\
\hline \multicolumn{3}{|c|}{ Dificuldade para andar mais de um quilômetro } \\
\hline Sem dificuldade & 40,63 & 68,48 \\
\hline Alguma dificuldade & 22,45 & 17,40 \\
\hline Grande dificuldade & 28,11 & 11,73 \\
\hline \multirow[t]{2}{*}{ Sem informação } & 8,81 & 2,39 \\
\hline & $p<0,0001$ & \\
\hline \multicolumn{3}{|c|}{ Dificuldade para andar cerca de cem metros } \\
\hline Sem dificuldade & 65,01 & 88,61 \\
\hline Alguma dificuldade & 17,06 & 7,72 \\
\hline Grande dificuldade & 9,12 & 1,85 \\
\hline \multirow[t]{2}{*}{ Sem informação } & 8,81 & 1,82 \\
\hline & $p<0,0001$ & \\
\hline
\end{tabular}

Fonte: PNAD 1998.

Valor p: teste Qui-quadrado de Pearson, com correção de Rao \& Scott.

As proporções levam em consideração o peso amostral dos indivíduos. Foram excluídos os indivíduos sem trabalho ou aposentadoria por representarem apenas $5 \%$ da população estudada. 
Tabela 4

Características sócio-demográficas associadas com a situação no mercado

de trabalho na população idosa masculina. Brasil, 1998

(categoria de referência $=$ aposentado).

Situação no mercado de trabalho

Trabalhando

OR (IC 95\%)

\begin{tabular}{|c|c|}
\hline \multicolumn{2}{|l|}{ Idade em anos } \\
\hline $65-69$ & 1,00 \\
\hline $70-74$ & $0,64(0,52-0,79)$ \\
\hline $75+$ & $0,23(0,19-0,28)$ \\
\hline \multicolumn{2}{|l|}{ Escolaridade em anos } \\
\hline $0-3$ & 1,00 \\
\hline $4-7$ & $0,81(0,65-1,02)$ \\
\hline $8-10$ & $0,91(0,69-1,20)$ \\
\hline$>11$ & $1,48(1,16-1,89)$ \\
\hline \multicolumn{2}{|c|}{ Pessoa de referência no domicílio } \\
\hline Sim & 1,00 \\
\hline Não & $0,55(0,43-0,70)$ \\
\hline \multicolumn{2}{|c|}{ Renda domiciliar per capita (em R\$) } \\
\hline$<130,00$ & 1,00 \\
\hline $130,00 \vdash 201,43$ & $1,09(0,83-1,44)$ \\
\hline $201,43 \vdash 341,00$ & $1,36(1,08-1,73)$ \\
\hline $341,00 \vdash 680,00$ & $1,13(0,83-1,55)$ \\
\hline$\geq 680,00$ & $2,24(1,44-3,49)$ \\
\hline \multicolumn{2}{|l|}{ Região de residência } \\
\hline Sudeste & 1,00 \\
\hline Sul & $1,40(1,14-1,71)$ \\
\hline Nordeste & $1,13(0,92-1,40)$ \\
\hline Norte & $1,05(0,72-1,54)$ \\
\hline Distrito Federal & $1,05(0,94-1,18)$ \\
\hline
\end{tabular}

Fonte: PNAD 1998

OR $=$ Odds ratio; IC = intervalo de confiança
Reino Unido em 1985 por Arber \& Ginn (1993), que correspondeu a $8 \%$ dos idosos do sexo masculino com 65 anos ou mais.

Em relação aos idosos que trabalhavam, verificamos o predomínio do trabalho informal, o que pode estar relacionado ao grande percentual dos idosos que já eram aposentados (73\%). $\mathrm{O}$ trabalho informal consiste, na maioria das vezes, no trabalho em ocupações precárias e com baixa remuneração. Segundo a Organização Pan-Americana de Saúde (OPAS, 2000), muitos idosos sustentam a si mesmos e a outros, trabalhando neste setor, especialmente nos países em desenvolvimento. Chama atenção o expressivo contingente de indivíduos que trabalhavam por conta própria. A tendência de migração de outras posições na ocupação para trabalho autônomo à medida que os trabalhadores envelhecem foi identificada em outros estudos (Ramos et al., 1997; Wajnman, 1995). A maior concentração de trabalho nos setores de serviço e industrial difere do relato de predomínio da atividade agrícola entre idosos brasileiros em 1996 (Camarano et al., 1999). Essa diferença, possivelmente se explica por este estudo incluir apenas os residentes em regiões metropolitanas.

Os idosos que permaneceram trabalhando eram mais jovens, possuíam maior renda domiciliar per capita, apresentavam maior escolaridade e eram referências domiciliares. Camarano et al. (1999) mostraram que a queda da taxa de atividade entre idosos no Brasil acentua-se com a idade, passando de $47 \%$ aos 65 anos de idade a 22,1\%, aos 75 anos. Outros estudos têm identificado que a maior escolaridade está associada com a permanência no mercado de trabalho em idades mais avançadas (Flippen \& Tienda, 2000; Parnes \& Sommers, 1994). Crystal et al. (1992) sugerem que a maior escolaridade afeta positivamente a renda dos idosos, dentre outras razões porque permite acesso ao trabalho mais qualificado e à continuidade da atividade laboral por mais tempo. Barros (1999) analisando a composição da renda de idosos pobres e não pobres, no Brasil, utilizando dados da PNAD de 1997, identifica que os rendimentos do trabalho constituem uma fração menor do rendimento domiciliar para os pobres do que para os não pobres e que o oposto ocorre em relação à aposentadoria. Esta diferença ressalta a importância da aposentadoria na elevação da renda das populações mais pobres, ou seja, na redução dos níveis de pobreza no país, pelo menos numa conjuntura de desemprego elevado e queda de salários.

Nossos achados são semelhantes aos dos estudos citados, sugerindo que desvantagens 
Indicadores de saúde e de utilização de serviços de saúde associados com a situação no mercado de trabalho na população idosa masculina. Brasil, 1998 (categoria de referência = aposentado).

\begin{tabular}{|c|c|c|}
\hline & \multicolumn{2}{|c|}{$\begin{array}{c}\text { Situação no mercado de trabalho } \\
\text { Trabalhando }\end{array}$} \\
\hline & OR (IC 95\%) & OR (IC 95\%) ajustada \\
\hline \multicolumn{3}{|c|}{ Percepção da própria saúde } \\
\hline Muito boa/boa & 1,00 & 1,00 \\
\hline Regular & $0,91(0,67-1,24)$ & $1,07(0,65-1,74)$ \\
\hline Muito ruim/ruim & $0,68(0,38-1,23)$ & $0,92(0,42-2,01)$ \\
\hline Outra pessoa & $1,23(0,85-1,76)$ & $1,61(0,97-2,66)$ \\
\hline \multicolumn{3}{|c|}{$\begin{array}{l}\text { Afastamento das atividades habituais } \\
\text { nas duas últimas semanas }\end{array}$} \\
\hline Não & 1,00 & 1,00 \\
\hline Sim & $0,62(0,42-0,93)$ & $0,68(0,42-1,11)$ \\
\hline \multicolumn{3}{|c|}{ Acamado nas duas últimas semanas } \\
\hline Não & 1,00 & 1,00 \\
\hline Sim & $0,69(0,44-1,09)$ & $0,77(0,48-1,25)$ \\
\hline \multicolumn{3}{|c|}{ Relato de doença crônica1 } \\
\hline Não & 1,00 & 1,00 \\
\hline Sim & $0,73(0,56-0,96)$ & $0,73(0,55-0,96)$ \\
\hline \multicolumn{3}{|c|}{ Plano de saúde privado } \\
\hline Não & 1,00 & 1,00 \\
\hline Sim & $1,12(0,79-1,60)$ & $0,75(0,55-1,00)$ \\
\hline \multicolumn{3}{|c|}{ Consulta médica nas duas últimas semanas } \\
\hline Não & 1,00 & 1,00 \\
\hline Sim & $0,86(0,60-1,24)$ & $0,80(0,61-1,04)$ \\
\hline \multicolumn{3}{|c|}{ Consulta odontológica nas duas últimas semanas } \\
\hline Não & 1,00 & 1,00 \\
\hline Sim & $4,58(1,73-12,13)$ & $2,35(0,46-12,12)$ \\
\hline \multicolumn{3}{|c|}{ Última visita ao dentista (anos) } \\
\hline$<1$ & 1,00 & 1,00 \\
\hline $1-2$ & $0,74(0,55-0,98)$ & $0,82(0,62-1,08)$ \\
\hline$>3$ & $0,52(0,42-0,66)$ & $0,81(0,55-1,20)$ \\
\hline Nunca & $0,42(0,23-0,78)$ & $0,60(0,34-1,05)$ \\
\hline \multicolumn{3}{|c|}{ Internação hospitalar no último ano } \\
\hline Não & 1,00 & 1,00 \\
\hline Sim & $0,80(0,60-1,06)$ & $0,79(0,52-1,21)$ \\
\hline
\end{tabular}

Fonte: PNAD 1998

OR = odds ratio; IC = intervalo de confiança.

OR (IC 95\%) ajustada = Odds ratio ajustada por idade, escolaridade, renda domiciliar per capita,

pessoa de referência no domicílio, região de residência, usando regressão logística múltipla.

Foram excluídos os indivíduos sem trabalho ou aposentadoria, que representaram apenas $5 \%$ da população estudada.

1 Doenças crônicas consideradas: artrite ou reumatismo, câncer, diabetes, bronquite ou asma, hipertensão,

doença do coração e cirrose. 
Indicadores autonomia e mobilidade física associados com a situação no mercado de trabalho na população idosa masculina. Brasil, 1998 (categoria de referência = aposentado).

\begin{tabular}{|c|c|c|}
\hline & \multicolumn{2}{|c|}{$\begin{array}{c}\text { Situação no mercado de trabalho } \\
\text { Trabalhando }\end{array}$} \\
\hline & OR (IC 95\%) & OR (IC 95\%) ajustada \\
\hline \multicolumn{3}{|c|}{$\begin{array}{l}\text { Dificuldade para alimentar-se, } \\
\text { tomar banho ou ir ao banheiro }\end{array}$} \\
\hline Sem dificuldade & 1,00 & 1,00 \\
\hline Alguma dificuldade & $0,40(0,22-0,72)$ & $0,52(0,27-0,99)$ \\
\hline Grande dificuldade & $0,11(0,05-0,25)$ & $0,15(0,07-0,33)$ \\
\hline \multicolumn{3}{|c|}{$\begin{array}{l}\text { Dificuldade para correr, levantar objeto pesado, } \\
\text { praticar esporte ou realizar trabalho pesado }\end{array}$} \\
\hline Sem dificuldade & 1,00 & 1,00 \\
\hline Alguma dificuldade & $0,66(0,54-0,80)$ & $0,68(0,55-0,84)$ \\
\hline Grande dificuldade & $0,52(0,41-0,64)$ & $0,53(0,44-0,64)$ \\
\hline \multicolumn{3}{|c|}{$\begin{array}{l}\text { Dificuldade para empurrar mesa } \\
\text { ou realizar conserto doméstico }\end{array}$} \\
\hline Sem dificuldade & 1,00 & 1,00 \\
\hline Alguma dificuldade & $0,55(0,42-0,72)$ & $0,59(0,45-0,78)$ \\
\hline Grande dificuldade & $0,34(0,24-0,48)$ & $0,41(0,28-0,60)$ \\
\hline \multicolumn{3}{|c|}{ Dificuldade para subir ladeira ou escada } \\
\hline Sem dificuldade & 1,00 & 1,00 \\
\hline Alguma dificuldade & $0,58(0,44-0,76)$ & $0,70(0,50-0,98)$ \\
\hline Grande dificuldade & $0,34(0,26-0,44)$ & $0,39(0,30-0,50)$ \\
\hline \multicolumn{3}{|c|}{ Dificuldade para abaixar-se, ajoelhar-se ou curvar-se } \\
\hline Grande dificuldade & 1,00 & 1,00 \\
\hline Alguma dificuldade & $0,62(0,45-0,86)$ & $0,66(0,47-0,91)$ \\
\hline Sem dificuldade & $0,32(0,23-0,43)$ & $0,37(0,27-0,52)$ \\
\hline \multicolumn{3}{|c|}{ Dificuldade para andar mais de um quilômetro } \\
\hline Grande dificuldade & 1,00 & 1,00 \\
\hline Alguma dificuldade & $0,68(0,49-0,94)$ & $0,81(0,56-1,17)$ \\
\hline Sem dificuldade & $0,28(0,21-0,37)$ & $0,32(0,24-0,44)$ \\
\hline \multicolumn{3}{|c|}{ Dificuldade para andar cerca de cem metros } \\
\hline Grande dificuldade & 1,00 & 1,00 \\
\hline Alguma dificuldade & $0,54(0,35-0,83)$ & $0,61(0,37-0,99)$ \\
\hline Sem dificuldade & $0,33(0,21-0,52)$ & $0,39(0,22-0,70)$ \\
\hline
\end{tabular}

Fonte: PNAD 1998.

$\mathrm{OR}=$ odds ratio; $\mathrm{IC}=$ intervalo de confiança.

OR (95\%) ajustada: Odds ratio (Intervalo de Confiança de 95\%) ajustada por idade, escolaridade, renda domiciliar per capita, pessoa de referência no domicílio e região de residência, usando regressão logística múltipla.

Foram excluídos os indivíduos sem trabalho ou aposentadoria por representarem apenas $5 \%$ da população estudada. 
estabelecidas em fases anteriores da vida continuam influenciando nas idades avançadas. Assim, os idosos com maior escolaridade, que provavelmente tiveram acesso a trabalhos mais qualificados, têm maior possibilidade de permanecer trabalhando, contribuindo para aumentar a renda domiciliar.

Com relação aos indicadores de saúde e de utilização de serviços de saúde estudados, os idosos que trabalhavam relataram menos doenças crônicas que os aposentados, confirmando que, também entre idosos, uma melhor condição de saúde está positivamente associada com o trabalho. A saúde é apontada não só como um dos principais determinantes para a permanência no trabalho, como também para o retorno ao trabalho após a aposentadoria em países desenvolvidos (Flippen \& Tienda, 2000). É interessante observar que, apesar do maior relato de doenças crônicas entre os aposentados, os dois grupos não diferiram com relação ao relato de internação hospitalar e consultas médicas, sugerindo diferenças no acesso a esses serviços.

A permanência na vida ativa parece estar determinada fortemente pela capacidade física. Essa idéia é reforçada pela forte associação do trabalho com melhores indicadores de autonomia e mobilidade física, mesmo após o ajuste pela idade e demais fatores sócio-demográficos. Nos Estados Unidos (Flippen \& Tienda, 2000), a limitação da capacidade física, em indivíduos com 51 a 61 anos, esteve associada a uma maior probabilidade de aposentadoria e desemprego e maior risco de sair do mercado de trabalho em dois anos de seguimento. Em certa medida, as desigualdades em saúde que observamos neste estudo podem ser explicadas, em parte, pela inclusão entre os aposentados daqueles que se aposentaram por motivo de saúde. Em 1998, das aposentadorias concedidas no Brasil pela previdência social, $1 \%$ foi por invalidez em pessoas acima de 65 anos (MPAS, 1999).

Nossos resultados confirmam que o efeito do trabalhador sadio (ETS) também está presente entre idosos, manifestando-se principalmente pela maior autonomia e mobilidade física dos que trabalhavam. Este efeito é atribuído ao fato de os indivíduos mais saudáveis terem maior chance de ingressar na força de trabalho (efeito de seleção) e também de permanecerem trabalhando (efeito de sobrevida) (Checkoway et al., 1989). Sua magnitude é geralmente medida através da menor mortalidade ao longo da vida e varia para diferentes categorias profissionais (Baillargeon, 1998; Chen \& Saetón, 1996; Meijers, 1989). Arber (1996), por exemplo, demonstrou que trabalhadores sem qualificação têm maior probabilidade de apresentar problemas de saúde do que os profissionais especializados. Nossos resultados sugerem que estudos ocupacionais em idosos necessitam considerar este efeito na interpretação de seus resultados sempre que usarem como referência a população geral ou uma população com composição distinta da que está sendo estudada.

Algumas considerações devem ser feitas com relação aos resultados desta análise. Este é um estudo transversal, há uma inter-relação entre as condições de saúde, as condições físicas e a situação no mercado de trabalho, cuja temporalidade só poderá ser captada em outros tipos de estudo. Um dos problemas relativos ao uso de dados de inquéritos é a mortalidade diferencial, os participantes são os que sobreviveram até o período do estudo, portanto tendem a ser mais saudáveis. Aqueles mais vulneráveis, com piores condições de vida e saúde, podem morrer mais cedo, e o trabalho está relacionado a uma maior sobrevida (Blanc et al., 1994). Mesmo considerando a possibilidade do viés de sobrevivência, nossos resultados demonstram que persistiram desigualdades nas condições de saúde dos idosos, segundo a situação no mercado de trabalho, salientando que essas estimativas podem ser conservadoras.

Neste inquérito, as informações foram obtidas através de entrevista domiciliar, havendo a possibilidade de uso de informante próximo ou equivalente. Em relação às medidas objetivas de saúde, estudos de confiabilidade têm demonstrado uma boa concordância entre informações prestadas pelo próprio entrevistado ou por respondente próximo (Armstrong et al., 1994). Não há razão para supor que os erros de classificação relativos ao uso do informante próximo em grandes inquéritos apresentem alguma tendenciosidade com o trabalho. Os erros de classificação existentes são, mais provavelmente, não diferenciais, tendendo a diluir as associações existentes.

Ao se utilizarem dados sobre morbidade referida, deve-se considerar a possibilidade de taxas de prevalência subestimadas devido ao viés de memória ou pela ausência de diagnóstico. Os indicadores de saúde e de utilização de serviços estudados restringiram-se ao período de duas semanas anteriores à pesquisa, exceto para a variável última visita ao dentista e internação hospitalar, o que tende a minimizar o viés de memória. Outro aspecto a ser considerado é que, na PNAD 1998, as perguntas relativas às doenças crônicas não especificam se as doenças foram diagnosticadas por um médico. Logo, não é possível excluir a possibilidade de que as respostas misturem informações de fontes va- 
riadas, tendo, assim, níveis de confiabilidade distintos.

Neste estudo, perdas mais importantes de informação (6\%) ocorreram para os indicadores de autonomia e mobilidade física, exceto para a pergunta dificuldade de alimentar-se, tomar banho ou ir ao banheiro. Segundo informação do IBGE, essas perdas correspondem à ausência deliberada de coleta da informação para os indivíduos que relataram grande dificuldade para realizar a atividade acima. As perdas foram significativamente mais freqüentes entre os idosos aposentados, com mais de 75 anos, menor escolaridade, menor renda domiciliar e que se afastaram mais das atividades habituais, estiveram mais tempo acamados, relataram mais doença crônica e internações no último ano (dados não mostrados). Tais perdas comprometem a estimativa das prevalências desses indicadores, subestimando a força das associações encontradas entre os mesmos e a situação no mercado de trabalho.

Em síntese, nossos resultados mostraram que um quarto dos idosos trabalhava e que a inserção no mercado de trabalho expressa di- ferenças relativas à idade, à renda domiciliar, à escolaridade e ao fato de ser referência domiciliar. Além disso, este estudo identificou que a melhor condição de saúde, em especial a preservação da autonomia e da mobilidade física, é um importante fator para a permanência na vida ativa nas idades mais avançadas. $\mathrm{O}$ envelhecimento populacional promove transformações profundas na sociedade, com mudanças na estrutura familiar, no mercado de trabalho e no perfil de demandas por políticas públicas, em especial nas áreas de saúde e seguridade social. Idosos com boas condições de saúde, com autonomia física e mental mantêm boas perspectivas de vida e podem assumir papéis relevantes na sociedade. Esta deve considerar a capacidade de trabalho dos idosos e garantir o direito à manutenção ou reinserção no mercado de trabalho daqueles que assim o desejarem. Como a capacidade de trabalho e a qualidade de vida dos idosos são determinadas, em grande parte, em etapas anteriores da vida, mudanças positivas na vida dos idosos significam, também, investimentos em educação, saúde e trabalho para os jovens e adultos de hoje.

\section{Agradecimentos}

Agradecemos ao Dr. Francisco Viacava, por gentilmente ter-nos repassado o algoritmo fornecido pela Fundação Instituto Brasileiro de Geografia e Estatística para corrigir o efeito do delineamento de amostragem da Pesquisa Nacional por Amostra de Domicílios de 1998; ao Dr. Henrique L. Guerra, pela valiosa ajuda quando da incorporação desta informação ao banco de dados; à Dra. Mônica Viegas Andrade, pela cuidadosa leitura e valiosas sugestões e à Drạ. Maria Fernanda Lima-Costa pelo apoio e incentivo durante a realização deste trabalho.

\section{Referências}

ARBER, S., 1987. Social class, non-employment, and chronic illness: Continuing the inequalities in health debate. BMJ, 294:1069-1073.

ARBER, S., 1996. Integrating nonemployment into research on health inequalities. International Journal of Health Services, 26:445-481.

ARBER, S. \& GINN, J., 1993. Gender and inequalities in health in later life. Social Science and Medicine, 36: 33-46.

ARMITAGE, P. \& BERRY, G., 1987. Statistical Methods in Medical Research. Oxford: Blackwell Scientific Publications. 
ARMSTRONG, B. K.; WHITE, E. \& SARACCI, R., 1994. Principles of Exposure Measurement in Epidemiolgy. Monographs on Epidemiology and Bioestatistics. Oxford: Oxford University Press.

BAILLARGEON, J.; WILKINSON, G.; RUDKIN, L.; BAILLARGEON, G. \& RAY, L., 1998. Characteristics of the healthy worker effect: A comparison of male and female occupatonal cahorts. Journal of Occupational and Environmental Medicine, 40:368-373.

BARROS, R. P.; MENDONÇA, R. \& SANTOS, D., 1999. Incidência e Natureza da Pobreza entre Idosos no Brasil. Texto para Discussão 686. Rio de Janeiro: Instituto de Pesquisa Econômica Aplicada.

BARRETO, S. M.; SWERDLOW, A. J.; SMITH, P. G.; HIGGINS, C. D. \& ANDRADE, A., 1996. Mortality from injuries and other causes in a cohort of 21.800 brasilian steel workers. Occupational and Environmental Medicine, 53:343-350.

BLANC, P. D.; KATZ, P. \& YELIN, E., 1994. Mortality risk among elderly workers. American Journal of Industrial Medicine, 26:543-547.

CAMARANO, A. A.; BELTRÃO, K. I.; PASCOM, A. R. P.; MEDEIROS, M.; CARNEIRO, I. G.; GOLDANI, A. M.; VASCONCELOS, A. M. N.; CHAGAS, A. M. R. \& OSÓRIO, R. G., 1999. Como vai o Idoso Brasileiro? Texto para Discussão 681. Rio de Janeiro: Instituto de Pesquisa Econômica Aplicada.

CARPENTER, L. M., 1990. Health-Related Selection and Mortality in Employees of the United Kingdom Atomic Energy Authority, 1946-79 and the Atomic Weapons Establishment, 1951-82. Ph.D. Thesis, London: London School of Hygiene and Tropical Medicine, University of London.

CHECKOWAY, H.; PEARCE, N. \& CRAWFORD-BROWN, D. J., 1989. Research Methods in Occupational Epidemiology. New York: Oxford University Press.

CHEN, R. \& SAETÓN, A., 1996. The influence of study characteristica on the healthy worker effect: A multiple regression analysis. Occupational Medicine, 46:345-350.

COSTA, G., 2000. Capacità di lavoro e invecchiamento. Medicina del Lavoro, 91:302-312.

CRISTAL, S.; SHEA, D. \& KRISHNASWAMI, S., 1992. Educational attainment, occupational history, and stratification: Determinants of later-life economic outcomes. Journal of Gerontology, 47:S213-S221.

FLIPPEN, C. \& TIENDA, M., 2000. Pathways to retirement: Patterns of labor force participation and labor market exit among the pre-retirement population by race, hispanic origin, and sex. Journal of Gerontology, 55B:S14-S27.

IBGE (Fundação Instituto Brasileiro de Geografia e Estatística), 1981. Censo Demográfico 1980. Rio de Janeiro: IBGE.

IBGE (Fundação Instituto Brasileiro de Geografia e Estatística), 2001. Censo Demográfico, 2000. Rio de Janeiro: IBGE

HOSMER, D. W. \& LAMENSHOW, S., 1989. Applied Logistic Regression. New York: John Wiley \& Sons.

JANLERT, U., 1997. Unemployment as a disease and disease of the unemployed. Scandinavian Journal of Work, Environment \& Health, 23:79-83.
LIMA-COSTA, M. F. F.; GUERRA, H. L.; BARRETO, S. M. \& MAIA, R., 2000. Diagnóstico da situação de saúde da população idosa brasileira: Um estudo da mortalidade e das internações hospitalares públicas. Informe Epidemiológico do SUS, 9:23-41.

MARMOT, M. G. \& SHIPLEY, M. J., 1996. Do socioeconomic differences in mortality persist after retirement? 25 years follow up of civil servants from the Whitehall study. BMJ, 313:1177-1180.

MEIJERS, J. M. M.; SWEN, G. M. H.; VOLOVICS, A.; LUCAS, L. J. \& van VLIET, K., 1989. Occupatonal cohort studies: The influence of design characteristics on the healthy worker effect. International Journal of Epidemiology, 18:970-975.

MPAS (Ministério da Previdência e Assistência Social), 1999. Anuário Estatístico da Previdência Social. Brasília: Instituto Nacional do Seguro Social, MPAS.

MOREIRA, M. M., 1998. O envelhecimento da população brasileira em nível regional; 1940-2050. In: XI Encontro Nacional de Estudos Populacionais, Anais, pp. 3030-3124, Caxambu: Associação Brasileira de Estudos Populacionais.

OPAS (Organização Pan-Americana da Saúde), 2000. Envelhecimento: Mitos na Berlinda. 19 Maio 2000 <http://www.opas.org.br/noticias/publicacoes/ Mitos\%20na\%Berlinda.htm>.

PARNES, H. S. \& SOMMERS, D. G., 1994. Shunning retirement: Work experience of men in their seventies and early eighties. Journal of Gerontology, 49: S117-S124.

PESSOA, D. G. C.; SILVA, P. L. N. \& DUARTE, R. P. N., 1997. Análise estatística de dados de pesquisas por amostragem: Problemas no uso de pacotes-padrão. Revista Brasileira de Estatística, 58:53-75.

RAMOS, L.; RIOS NETO, E. \&WAJNMAN, S., 1997. Perfis etários, posição na ocupação e desigualdade de rendimentos no Brasil. Nova Economia, 7:11-41.

SCHKOLNIK, S., 1989. El envejecimiento de la poblacion de América Latina 19950-2025. In: El Proceso de Envejecimiento de la Poblacion (J.-C. Chesnais, org.), pp. 104-145, Santiago: Comisión Económica para América Latina y el Caribe.

SCHLESSELMAN, J. J., 1982. Case-Control Studies. Design, Conduct Análysis. New York: Oxford University Press.

TRUELSEN, T.; BONITA, R. \& JAMROZIK, K., 2001. Survillance of stroke: A global perspective. International Journal of Epidemiology, 30:S11-S12.

WAJNMAN, S., 1997. Tendências Prospectivas de Crescimento da População Economicamente Ativa no Brasil. Textos para Discussão 111. Belo Horizonte: Centro de Desenvolvimento e Planejamento Regional, Faculdade de Ciências Econômicas, Universidade Federal de Minas Gerais.

WAJNMAN, S., 1995. O Padrão da Mobilidade Ocupacional do Ciclo de Vida Masculino no Brasil. Texto para Discussão. Rio de Janeiro: Instituto de Pesquisa Econômica Aplicada.

Recebido em 6 de maio de 2002

Aprovado em 1 de julho de 2002 